\title{
Navier-Stokes Equations and Area of Interfaces
}

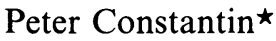 \\ Department of Mathematics, University of Chicago, 5734 University Avenue, Chicago, IL 60637, USA
}

Abstract. We present new a priori estimates for the vorticity of solutions of the three dimensional Navier-Stokes equations. These estimates imply that the $L^{1}$ norm of the vorticity is a priori bounded in time and that the time average of the $4 /(3+\varepsilon)$ power of the $L^{4 /(3+\varepsilon)}$ spatial norm of the gradient of the vorticity is a priori bounded. Using these bounds we construct global Leray weak solutions of the Navier-Stokes equations which satisfy these inequalities. In particular it follows that vortex sheet, vortex line and even more general vortex structures with arbitrarily large vortex strengths are initial data which give rise to global weak solutions of this type of the Navier-Stokes equations. Next we apply these inequalities in conjunction with geometric measure theoretical arguments to study the two dimensional Hausdorff measure of level sets of the vorticity magnitude. We obtain a priori bounds on an average such measure, $\langle\mu\rangle$. When expressed in terms of the Reynolds number and the Kolmogorov dissipation length $\eta$, these bounds are

$$
\langle\mu\rangle \leqq \frac{L^{3}}{\eta}\left(1+\mathrm{Re}^{-1 / 2}\right)^{1 / 2} .
$$

The right-hand side of this inequality has a simple geometrical interpretation: it represents the area of a union of non-overlapping spheres of radii $\eta$ which fill a fraction of the spatial domain. As the Reynolds number increases, this fraction decreases. We study also the area of level sets of scalars and in particular isotherms in Rayleigh-Benard convection. We define a quantity, $\langle\mu\rangle_{r, t}\left(x_{0}\right)$ describing an average value of the area of a portion of a level set contained in a small ball of radius $r$ about the point $x_{0}$. We obtain the inequality

$$
\langle\mu\rangle_{r, t}\left(x_{0}\right) \leqq C \kappa^{-1 / 2} r^{5 / 2}\left\langle v\left(x_{0}\right)\right\rangle^{1 / 2},
$$

where $\kappa$ is the diffusivity constant and $\langle v\rangle$ is the velocity. The inequality is valid for $r$ small but larger than a small scale $\lambda=\kappa\langle v\rangle^{-1}$. In the case of turbulent velocities this scale is smaller than the smallest significant physical scale,

* Partially supported by NSF grant DMS-860-2031. Sloan Research Fellow 
suggesting that 2.5 is a lower bound for the fractal dimension of an (ensemble) average interface in turbulent flow, a fact which agrees with the experimental lower bound of 2.35 .

We define a similar quantity $\langle\mu\rangle_{\delta, t}$ representing an average value of the area of a portion of a level set contained in the region of space $D_{\delta}=\{x \in D ; \operatorname{dist}(x, \partial D)>\delta\}$, where $D$ is the domain of aspect ratio of order one and diameter $L$, where the convection takes place. We obtain the inequality

$$
\langle\mu\rangle_{\delta, t} \leqq C\left(L^{3} \delta^{-1}+L^{5 / 2} \delta^{-1 / 2} \mathrm{Ra}^{1 / 2}\right),
$$

where $\mathrm{Ra}$ is the Rayleigh number.

\section{Introduction}

The mathematical theory of the three dimensional incompressible Navier-Stokes equations remains dominated by the result of Leray ([L]) published in 1934 which established the existence of global weak solutions for arbitrary initial data with finite energy. The basic property of these weak solutions is that they satisfy an energy inequality

$$
\frac{1}{2} \int|u(x, t)|^{2} d x+v \int_{0}^{t} \int|\nabla u(x, s)|^{2} d x d s \leqq \frac{1}{2} \int\left|u_{0}(x)\right|^{2} d x+\int_{0}^{t} \int F(x, s) \cdot u(x, s) d x d s .
$$

In (1.1) we denote by $u$ the three-component, divergence-free velocity vector, by $v$ the kinematic viscosity coefficient, $u_{0}$ the initial velocities, and by $F$, the body forces. We assume periodic boundary conditions. The spatial integrals are taken over a standard cube of periodicity $[-L / 2, L / 2]^{3}$. It follows from (1.1) that the space and time integral of the square of the magnitude of gradient of the velocities is a well-defined quantity, bounded a priori in terms of the initial data, $F$ and, of course $v$. The vorticity, $\omega=\operatorname{curl} u$ satisfies the equation

$$
\left(\frac{\partial}{\partial t}+u \cdot \nabla-v \Delta\right) \omega=\omega \cdot \nabla u+f
$$

where $f=\operatorname{curl} F$. In view of the energy inequality, the right-hand side of (1.2) is a priori bounded in $L^{1}$ in space and time provided $F$ and $f$ are sufficiently regular. This suggests that

$$
\sup _{0 \leqq t} \int|\omega(x, t)| d x
$$

is a priori bounded if the initial vorticity is in $L^{1}$ in space. The proof of this fact and related matters are the subject of Sect. 2 of the present work. The main technical device is the use of the change of dependent variable

$$
w(x, t)=q(\tau \omega(x, t)) .
$$

In (1.3) $\tau$ is an arbitrary positive constant which has the dimension of time, and $q$ is a smooth positive function of the dimensionless variable $y \in \mathbb{R}^{3}$, which has bounded gradient and positive definite Hessian. Because its gradient is bounded, 
the Hessian of $q$ must decay, for large $y$, at least as $|y|^{-1}$ does. In our arguments we use specific choices of $q$ (see (2.16)). The equation satisfied by $w$ resembles the one satisfied by $\omega$ and direct integration provides the sought a priori information on $\omega$ and on $\nabla \omega$, at least for smooth solutions. The requirement that the solution be smooth is not necessary, however. We consider initial data such that the velocity $u_{0}$ is square integrable and divergence-free and such that the vorticity $\omega_{0}$ is a finite Radon measure on the torus. Expressed in terms of the initial vorticity alone, these conditions are first, that $\omega_{0}$ is a finite divergence-free Radon measure on the torus and secondly, that $\omega_{0}$ belongs also to the space $V^{\prime} . V^{\prime}$ is the dual of the space $V$ of square integrable, divergence-free functions on the torus whose first order derivatives are periodic and square integrable. Under these assumptions, together with appropriate regularity requirements on $F, f$ we prove that the Navier-Stokes equations possess global Leray weak solutions for which

$$
\underset{0 \leqq t}{\operatorname{ess.sup}} \int|\omega(x, t)| d x
$$

and

$$
\int_{0}^{t} \int|\nabla \omega(x, s)|^{4 /(3+\varepsilon)} d x d s
$$

are a priori bounded in terms of the data (Theorem 2.2). The proof of this fact uses the change of dependent variable (1.3) for the vorticity of certain approximate solutions. The approximation procedure we chose is the one employed in $[\mathrm{C}-\mathrm{K}-\mathrm{N}]$ for the construction of suitable weak solutions (that is, solutions which satisfy a local energy estimate). The reason for this choice is the fact that these approximate solutions obey a vorticity equation sufficiently similar to (1.2) for our method to succeed. Incidentally, we obtain thus suitable weak solutions and therefore the 1-dimensional Hausdorff measure of their space and time singular set is zero $([\mathrm{C}-\mathrm{K}-\mathrm{N}])$. The class of initial vorticities for which our result is applicable contains the physically interesting examples of vortex sheets and vortex lines. We recall that there are results in the literature concerning the case of small initial data. For instance, in $[\mathbf{G}-\mathbf{M}]$ it is shown that if $\omega_{0}$ belongs to the Morrey class $\frac{3}{2}$ and is small then there exist unique, globally smooth solutions which decay to zero (the result is for $F=0$ ). Similar results are stated in $[\mathrm{C}-\mathrm{S}]$. In our case, large data are permitted, allowing for complex dynamical behavior. Moreover, dimensional analysis suggests that measures which are concentrated on certain sets of Hausdorff dimension $\geqq \frac{1}{2}$ and are smooth with respect to Hausdorff measure, give rise to vortex structures for which our results are applicable.

Theorem 2.2 is applicable for initial velocities which are square integrable, divergence-free and of bounded variation. However, we do not know whether $\int|\nabla u(x, t)| d x$ is bounded a priori in time for general data. What we can prove is that there exists an absolute constant $c$ such that

$$
\int_{0}^{t} \exp \left(c \int|\nabla u(x, s)| d x\right) d s
$$

is bounded a priori in terms of the data. The proof of this bound uses the fact that 
the map $\omega \rightarrow \nabla u$ is of weak-type $(1,1)$ and the fact $[F-G-T]$ that

$$
\int_{0}^{t} d s\|\nabla u(\cdot, s)\|_{L^{\infty}}^{1 / 2}
$$

is a priori bounded.

The results of $[\mathrm{F}-\mathrm{G}-\mathrm{T}]$ (see also [D] for the case of bounded domains) are the only ones that we are aware of, in which the behavior of high order derivatives for weak solutions is investigated. For instance, it is proven in $[\mathrm{F}-\mathrm{G}-\mathrm{T}]$ that

$$
\int_{0}^{t} d s\left(\int|\Delta u(x, s)|^{2} d x\right)^{1 / 3}
$$

is a priori bounded in terms of the initial data, for arbitrary $L^{2}$ data. Our corresponding result is the boundedness of

$$
\int_{0}^{t} \int|\Delta u(x, s)|^{4 /(3+\varepsilon)} d x d s
$$

Neither result implies the other and neither seems sufficient for global regularity. It may be useful to mention here that a sufficient condition for global regularity is the boundedness of

$$
\int_{0}^{t} d s\left(\int|\Delta u(x, s)|^{2} d x\right)^{2 / 3}
$$

(see [C-F]. A simple modification of the argument on p. 79 yields the result above).

In Sect. 3 we consider the problem of estimating a priori, in terms of the Reynolds number, the average area of a level set of the vorticity magnitude. Thus, we consider the level sets

$$
A_{\gamma}=\{x ;|\omega(x, t)|=\gamma\} .
$$

If $\omega$ is smooth, a well-known result ([M]) states that for almost all values of $\gamma$ the sets $A_{y}$ are smooth 2-dimensional manifolds without boundary on the torus. We denote their 2-dimensional Hausdorff measure (area in this case) by

$$
\mu(\gamma, t)=\int_{A_{\gamma}} d H^{(2)}(x),
$$

and the time average of this quantity by

$$
\mu_{T}(\gamma)=T^{-1} \int_{0}^{T} \mu(\gamma, t) d t
$$

The main result of Sect. 3 is an a priori bound on

$$
\int_{0}^{\infty} \mu_{T}^{2}(\gamma) d \gamma
$$

in terms of the data (Theorem 3.2). In the particular case of $F=0$ (corresponding to decaying turbulence) this a priori bound can be written in terms of the Kolmogorov dissipation length $\eta$ and the Reynolds number Re. It states that the 
average

$$
\langle\mu\rangle_{T}=\left(\gamma_{\max }^{-1} \int_{0}^{\gamma_{\max }} \mu_{T}^{2}(\gamma) d \gamma\right)^{1 / 2}
$$

satisfies the inequality

$$
\langle\mu\rangle_{T} \leqq \frac{L^{3}}{\eta}\left(1+\mathrm{Re}^{-1 / 2}\right)^{1 / 2} .
$$

We recall that the Kolmogorov length is defined in terms of the average dissipation rate $\varepsilon$ :

$$
\eta=\varepsilon^{-1 / 4} v^{3 / 4}
$$

The relation between $T, \operatorname{Re}$ and the average energy dissipation rate $\varepsilon$ is the one appropriate for decaying turbulence $([\mathrm{B}])$,

$$
\operatorname{Re}=T^{2} \varepsilon v^{-1}
$$

that is, $T$ represents a time scale large enough for the turbulence to display universal statistical features at small scales but shorter than the time scale $L^{2} v^{-1}$ of viscous decay.

The main technical tools are a new change of dependent variable and a geometric measure theoretical lemma. The change of dependent variable is performed on the variable $w$ used in Sect. 2 (see (1.3)). It introduces the function $g$ defined by

$$
g(x, t)=G(w(x, t))
$$

where the smooth, increasing, positive, convex function of one variable $G$ is defined by

$$
G(\beta)=\int_{0}^{\beta}(\beta-\lambda) \phi^{2}(\lambda) d \lambda
$$

with $\phi$ an arbitrary smooth function supported in $\left(\tau^{-1}, \infty\right)$. The fact that $\phi$ is arbitrary plays an important role. The properties of $G$ and the equation satisfied by $g$ are such that the integral

$$
\int_{0}^{t} \int \phi^{2}(w(x, s))|\nabla w(x, s)|^{2} d x d s
$$

is bounded a priori in terms of the data, for all $t$. This integral bounds the square of the scalar product in $L^{2}\left(\mathbb{R}_{+}\right)$of $\mu_{T}$ and $\phi$. The main fact which enables us to prove the last statement is an important lemma used in geometric measure theory $([\mathrm{K}],[\mathrm{F}])$. It establishes the formula

$$
\int \chi(x)|\nabla w(x)| d x=\int_{0}^{\infty} d \beta \int_{\{x ;|w(x)|=\beta\}} \chi(x) d H^{(n-1)}(x)
$$

for arbitrary Borel measurable nonnegative functions $\chi$ supported in some open set of $\mathbb{R}^{n}$ and arbitrary Lipschitz continuous functions $w$ in that set. In (1.6) $H^{(n-1)}$ is $n-1$-dimensional Hausdorff measure. We believe that (1.6) is important, not only 
because it is suitable for analysis, but also because it permits the practical computation of the area of level sets without the need to track them. Let $\psi$ be a smooth nonnegative function equal to one in some region of $\mathbb{R}^{n}$ and with compact support. A consequence of (1.6) is the identity

$$
\int_{0}^{\infty} \phi(\beta) \mu_{\psi}(\beta) d \beta=\int \psi(x) \phi(w(x))|\nabla w(x)| d x,
$$

where

$$
\mu_{\psi}(\beta)=\int_{\{x:|w(x)|=\beta\}} \psi(x) d H^{(n-1)}(x) .
$$

Taking $\phi(\beta)=\exp (-i k \beta)$ in (1.7) one finds the Fourier transform of $\mu_{\psi}$ and taking a standard smooth mollifier for $\phi$ one approximates the individual values of $\mu_{\psi}$. These objects are computed from a general knowledge of $w$ but without the need to resolve delicate small scale details.

Section 4 is devoted to the study of level sets of scalars and, in particular, to isotherms in turbulent Rayleigh-Benard convection. The scalar $T$ satisfies the equation

$$
\left(\frac{\partial}{\partial t}+u \cdot \nabla-\kappa \Delta\right) T=0
$$

in an open bounded set $D$ with smooth boundary $\partial D$ included in $\mathbb{R}^{3}$. The time dependent function $u$ is assumed to vanish on $\partial D$ and be divergence-free. The Eq. (1.9) is supplemented with initial data and boundary conditions which imply a maximum principle

$$
0 \leqq T(x, t) \leqq M,
$$

valid for all $x, t$. Well-posedness of (1.9) and the bound (1.10) are the only consequences of the boundary conditions on $T$ used in this work. Here is a rapid proof of the maximum principle for (1.9) in the case of homogeneous Dirichlet, Neumann or periodic boundary conditions. We do not assume, for this proof either that $T$ is scalar, or that $u$ is divergence free. We require that either the normal component of $u$ vanishes on the boundary or, if $T$ is periodic that $u$ be periodic.

Let $F(\lambda)=\exp (r \lambda)$, where $r>0$ is arbitrary. The function $h(x, t)=F\left(|T(x, t)|^{2}\right)$ satisfies

$$
\left(\frac{\partial}{\partial t}+u \cdot \nabla-\kappa \Delta\right) h+2 \kappa r h|\nabla T|^{2}+\left.\left.\kappa r^{2} h|\nabla| T\right|^{2}\right|^{2}=0 .
$$

Integrating in (1.11) and using the boundary conditions we deduce

$$
\frac{d}{d t} \int h(x, t) d x \leqq\|\operatorname{div} u(\cdot, t)\|_{L^{\infty}} \int h(x, t) d x,
$$

and from Gronwall's inequality

$$
\int h(x, t) d x \leqq\left(\int h(x, 0) d x\right) \exp \left(\int_{0}^{t}\|\operatorname{div} u(\cdot, s)\|_{L^{\infty}} d s\right) .
$$


Taking the $r^{\text {th }}$ root of (1.12) and then letting $r \rightarrow \infty$, we get

$$
\left\|\exp \left(|T(\cdot, t)|^{2}\right)\right\|_{L^{\infty}} \leqq\left\|\exp \left(|T(\cdot, 0)|^{2}\right)\right\|_{L^{\infty}}
$$

and taking the logarithm

$$
\|T(\cdot, t)\|_{L^{\infty}} \leqq\|T(\cdot, 0)\|_{L^{\infty}} .
$$

The proof above can be used to prove that the 2 or 3 dimensional Burgers equation

$$
\left(\frac{\partial}{\partial t}+u \cdot \nabla-v \Delta\right) u+\nabla p\left(|u|^{2}\right)=0
$$

has global smooth solutions for arbitrarily large initial data.

We return to the scalar equation (1.9). The formula (1.8) is used, with appropriate $\psi$ with compact support in $D$, to study the areas of portions of level sets of $T$ which are situated at a positive distance from $\partial D$. The change of dependent variable used for this prupose is

$$
g_{\psi}(x, t)=\psi(x) G(T(x, t))
$$

with $G$ defined in (1.5).

For any fixed $x_{0}$ in $D$ we obtain the bound

$$
\langle\mu\rangle_{r, t}\left(x_{0}\right) \leqq C \kappa^{-1 / 2} r^{5 / 2}\left\langle v\left(x_{0}\right)\right\rangle^{1 / 2},
$$

where $\langle\mu\rangle_{r, t}\left(x_{0}\right)$ represents an average area of a piece of isotherm situated in the ball of radius $r$ centered at $x_{0}$ and $\left\langle v\left(x_{0}\right)\right\rangle$ is an average velocity (see (4.17)-(4.30)). The inequality (1.15) is valid for small $r$ but $r$ is not allowed to become vanishingly small. Rather $r \geqq \lambda$, where

$$
\lambda=\kappa\langle v\rangle^{-1}
$$

is a local scale. In the case of turbulent velocities, the scale is at least as small as the smallest physically significant scale. For instance, if $u$ has the statistics given by a homogeneous self-similar family of solutions of the Navier-Stokes equations, then $\lambda=\operatorname{Pr}^{-1} \operatorname{Re}^{-1 / 4} \eta$ with $\operatorname{Pr}=v / \kappa$ and $\eta$ the Kolmogorov scale ([Fo]). In the case of Rayleigh-Benard convection

$$
\lambda=L \mathrm{Ra}^{-1+2 \beta},
$$

where $L$ is the outer scale, $\mathrm{Ra}$ is the Rayleigh number and $L \mathrm{Ra}^{-\beta}$ is the width of the thermal boundary layer. Thus $\lambda$ is equal to this width in the classical $\left(\beta=\frac{1}{3}\right)$ case and smaller than this width in the so-called hard turbulence $\left(\beta=\frac{2}{7}\right)$ case (see [Chi]). If $r$ is smaller than $\lambda$ then instead of (1.15) an $r^{2}$ behavior occurs, reflecting the fact that the surfaces are mathematically smooth if one is allowed to pass to arbitrarily small scales. The power 2.5 of $r$ which appears in (1.15) agrees with the experimental evidence ( $[\mathrm{S}]$ ) which seems to indicate that turbulent interfaces have a Hausdorff dimension larger or equal to 2.35. Although (4.15) is proven for Eq. (1.9) a similar computation can be made for the level sets of the vorticity magnitude. Then (4.15) would not be inconsistent with results obtained by numerical investigations of models of turbulence (see [Cho] and references therein). 


\section{Bounds on the Vorticity}

We consider the Navier-Stokes equation

$$
\frac{\partial u}{\partial t}+u \cdot \nabla u-v \Delta u+\nabla p=F
$$

in three dimensions, with periodic boundary conditions

$$
u\left(x+L e_{j}, t\right)=u(x, t)
$$

where $e_{j}=\left(\delta_{j k}\right), j, k=1,2,3$ is the standard basis in $\mathbb{R}^{3}$,

$$
\operatorname{div} u=0
$$

and initial conditions

$$
u(x, 0)=u_{0}(x)
$$

The vorticity, $\omega=\operatorname{curl} u$ satisfies the equation

$$
\frac{\partial \omega}{\partial t}+u \cdot \nabla \omega-v \Delta \omega=\omega \cdot \nabla u+f
$$

where $f=\operatorname{curl} F$. The main results of this section are a priori estimates for the vorticity in $L^{1}$ and certain estimates regarding the gradient of the vorticity. We start by explaining the heart of the matter, which is the following observation. The evolution of the square of the magnitude of the vorticity $|\omega|^{2}=\left(\omega_{1}\right)^{2}+\left(\omega_{2}\right)^{2}+\left(\omega_{3}\right)^{2}$ is given by

$$
\frac{1}{2}\left(\frac{\partial}{\partial t}+u \cdot \nabla-v \Delta\right)|\omega|^{2}+v|\nabla \omega|^{2}=(\omega \cdot \nabla u) \omega+f \omega .
$$

Considering the function $v(x, t)=|\omega(x, t)|$ we note that

$$
-\Delta v^{2}=2\left(-v \Delta v-|\nabla v|^{2}\right)
$$

and that

$$
|\nabla v|^{2} \leqq|\nabla \omega|^{2}
$$

Dividing in (2.6) by $|\omega|$ we obtain

$$
\frac{\partial v}{\partial t}+u \cdot \nabla v-v \Delta v \leqq|\nabla u||\omega|+|f| .
$$

Because the right-hand side of (2.7) belongs to $L^{1}$ in space and time and its integral is a priori bounded by quantities involving only the spatial $L^{2}$ norm of the initial data it follows, formally at least, that the $L^{1}$ spatial norm of the vorticity is bounded in time if it is finite initially. We will make the preceding argument rigorous and extend it because information is lost dividing by $|\omega|$ in (2.6). To clarify this let us consider an arbitrary smooth function

$$
q: \mathbb{R}^{3} \rightarrow \mathbb{R}
$$

multiply the $i^{\text {th }}$ equation (2.5) by $\tau\left(\partial q / \partial y_{i}\right)$ computed at $y=\tau \omega(x, t)$ and add. The 
constant $\tau$ is an arbitrary positive constant which has the dimension of time so that $y$ is dimensionless. It follows that the scalar dimensionless function

$$
w(x, t)=q(\tau \omega(x, t))
$$

satisfies the equation

$$
\left(\frac{\partial}{\partial t}+u \cdot \nabla-v \Delta\right) w+v \tau^{2} \frac{\partial^{2} q}{\partial y_{i} \partial y_{l}} \frac{\partial \omega_{i}}{\partial x_{j}} \frac{\partial \omega_{l}}{\partial x_{j}}=R
$$

with

$$
R=\tau \omega_{j} \frac{\partial u_{i}}{\partial x_{j}} \frac{\partial q}{\partial y_{i}}+\tau f_{i} \frac{\partial q}{\partial y_{i}}
$$

Now, clearly, if the function $q$ has bounded gradient

$$
|\nabla q| \leqq C
$$

uniformly for all $y$ in $\mathbb{R}^{3}$ then

$$
|R| \leqq \tau C(|\omega||\nabla u|+|f|)
$$

and thus $R$ is a priori integrable in space and time. One can choose $q$ such that $|\nabla q|$ is bounded and also $q$ is strictly convex. In view of the mean value theorem one sees that this is possible only if the Hessian of $q$ decays at least as does $|y|^{-1}$ for $y$ large. If we seek $q$ of the form

$$
q=h\left(1+|y|^{2}\right)
$$

we deduce

$$
\frac{\partial^{2} q}{\partial y_{i} \partial y_{j}} \xi_{i} \xi_{j}=2\left[\left|\xi_{\perp}\right|^{2} h^{\prime}(\alpha)+\left|\xi_{\mid}\right|^{2}\left(h^{\prime}(\alpha)+(2 \alpha-2) h^{\prime \prime}(\alpha)\right)\right],
$$

where $\alpha=1+|y|^{2}$ and $\xi_{\|}, \xi_{1}$, are the decomposition of the arbitrary vector $\xi$ in vectors parallel to and perpendicular to $y$. Consequently, any bounded, positive, increasing function of one variable $\eta$ determines $h$ and hence $q$ by the formula

$$
h^{\prime}(\alpha)=\frac{\eta(\alpha)}{(2 \alpha-2)^{1 / 2}} \text {. }
$$

It is clear that the slower the decay of $\eta^{\prime}$, the closer to optimal $q$ will be. For instance, a choice of $\eta$ given by

$$
\eta^{\prime}=\alpha^{-1}(\log (1+\alpha))^{-2}
$$

for large $\alpha$ provides a $q$ whose Hessian is bounded below by a multiple of

$$
\left(1+|y|^{2}\right)^{-1 / 2}\left(\log \left(2+|y|^{2}\right)\right)^{-2} .
$$

Because we intend to use $L^{p}$ spaces we will content ourselves with a choice of $q$ given by

$$
q(y)=\left(1+|y|^{2}\right)^{1 / 2}-\frac{1}{2(1-\varepsilon)}\left(1+|y|^{2}\right)^{(1-\varepsilon) / 2}
$$


for any positive $\varepsilon \leqq \frac{1}{2}$. It follows by direct computation of (2.14) that

$$
\frac{\partial^{2} q}{\partial y_{i} \partial y_{j}} \xi_{i} \xi_{j}>\frac{\varepsilon}{2}\left(1+|y|^{2}\right)^{-(1+\varepsilon) / 2}|\xi|^{2}
$$

Also,

$$
|\nabla q| \leqq 1
$$

and, cearly,

$$
\frac{1-2 \varepsilon}{2(1-\varepsilon)}\left(1+|y|^{2}\right)^{1 / 2} \leqq q \leqq\left(1+|y|^{2}\right)^{1 / 2} .
$$

With this choice of $q$ it follows from (2.8)-(2.19) that any classical solution of the Navier-Stokes equation satisfies

$$
\begin{aligned}
& \frac{1-2 \varepsilon}{2(1-\varepsilon)} \int\left(1+\tau^{2}|\omega(x, t)|^{2}\right)^{1 / 2} d x \\
& \quad+\frac{\varepsilon}{2} \nu \tau^{2} \int_{0}^{t} \int\left(1+\tau^{2}|\omega(x, s)|^{2}\right)^{-(1+\varepsilon) / 2}|\nabla \omega(x, s)|^{2} d x d s \leqq \gamma,
\end{aligned}
$$

where $\gamma$ is given by

$$
\gamma=\tau \int_{0}^{t} \int|\nabla u(x, s)||\omega(x, s)| d x d s+\tau \int_{0}^{t} \int|f(x, s)| d x d s+\int\left(1+\tau^{2}\left|\omega_{0}(x)\right|^{2}\right)^{1 / 2} d x .
$$

As it is well known, $\gamma$ is a priori bounded because of the energy inequality

$$
\int_{0}^{t} \int|\nabla u(x, s)|^{2} d x d s \leqq v^{-1} \int\left|u_{0}(x)\right|^{2} d x+L^{2} v^{-2} \int_{0}^{t} \int|F(x, s)|^{2} d x d s .
$$

The spatial integrals above are on a standard period box, $[-L / 2, L / 2]^{3}$. In view of the energy inequality it follows from (2.20) that

$$
\int_{0}^{t} \int|\nabla \omega(x, s)|^{4 /(3+\varepsilon)} d x d s
$$

is a priori bounded, for all $t$ positive. Indeed, we write

$$
|\nabla \omega(x, s)|^{4 /(3+\varepsilon)}=\left(|\nabla \omega(x, s)|^{4 /(3+\varepsilon)}\left(1+\tau^{2}|\omega(x, s)|^{2}\right)^{-(1+\varepsilon) /(3+\varepsilon)}\right)\left(\left(1+\tau^{2}|\omega(x, s)|^{2}\right)^{(1+\varepsilon) /(3+\varepsilon)}\right)
$$

and use a Hölder inequality in the space and time integral raising the first parenthesis to $(3+\varepsilon) / 2$ and the second one to the conjugate power, $(3+\varepsilon) /(1+\varepsilon)$. We obtain

$$
\int_{0}^{t} \int|\nabla \omega(x, s)|^{4 /(3+\varepsilon)} d x d s \leqq\left(2 \gamma \varepsilon^{-1} v^{-1} \tau^{-2}\right)^{2 /(3+\varepsilon)}\left(\int_{0}^{t} \int\left(1+\tau^{2}|\omega(x, s)|^{2}\right) d x d s\right)^{(1+\varepsilon) /(3+\varepsilon)}
$$

We proved

Theorem 2.1. Let $u$ be a classical $\left(\mathscr{C}^{3}\right)$ L-periodic solution of the three dimensional Navier-Stokes equation (2.1) with smooth periodic driving forces $F$. Assume that $u$ is defined for $0 \leqq t \leqq T$, where $T \leqq \infty$. Then, for every $\varepsilon, 0<\varepsilon \leqq \frac{1}{2}$ and $\tau, \tau>0$ the 
inequalities (2.20), (2.21), (2.22) hold for all $t<T$. In particular

and

$$
\sup _{0 \leqq t \leqq T} \int|\omega(x, t)| d x
$$

$$
\int_{0}^{t} \int|\nabla \omega(x, s)|^{4 /(3+\varepsilon)} d x d s
$$

are bounded in terms of the initial data and $F$.

In view of the Calderon-Zygmund inequality

$$
\int|\nabla v(x)|^{p} d x \leqq C_{p} \int|\operatorname{curl} v(x)|^{p} d x
$$

valid for divergence free functions and $1<p<\infty$ it follows from (2.22) that

$$
\int_{0}^{t} \int|\nabla \nabla u(x, s)|^{4 /(3+\varepsilon)} d x d s
$$

is bounded a priori in terms of the data. On the other hand, the a priori boundedness of

$$
\int|\nabla u(x, t)| d x
$$

does not follow from the preceding considerations. If one tries to imitate the proof of boundedness of the spatial $L^{1}$ norm of the vorticity for the full gradient one encounters the difficulty that the Hessian of the pressure is not estimated a priori in $L^{1}$ (space and time) in terms of the data. Of course these remarks concern the general case of large data and large $T$. The restriction to classical solutions is not necessary:

Theorem 2.2. Let $T>0, L>0, v>0$ be arbitrary. Assume that the initial data $u_{0}$ is a square integrable, L-periodic, divergence free $\mathbb{R}^{3}$ valued function whose curl, $\omega_{0}$ is a finite, signed, regular, vectorial Borel measure on the three dimensional torus $T_{L}^{3}=\mathbb{R}^{3} / L \mathbb{Z}^{3}$. Assume that the body forces $F$ are $L$ periodic, their curl, $f$ is $L$ periodic and they satisfy

and

$$
\int_{0}^{T} \int|F(x, t)|^{2} d x d t<\infty
$$

$$
\int_{0}^{T} \int|f(x, t)| d x d t<\infty
$$

Then there exists a Leray weak solution $u$ of the Navier-Stokes equations (2.1)-(2.4) whose curl (in the sense of distributions) belongs, for almost all $t$ (in the sense of Lebesgue measure), to $L^{2}$ on the torus $T_{L}^{3}$ and whose $L^{1}$ norm is uniformly bounded,

$$
\text { ess.sup } \int|\omega(x, t)| d x<\infty .
$$

Moreover, for every $0<\varepsilon \leqq \frac{1}{2}$,

$$
\int_{0}^{T} \int|\nabla \omega(x, s)|^{4 /(3+\varepsilon)} d x d s<\infty,
$$


and, consequently

$$
\int_{0}^{T} \int|\nabla \nabla u(x, s)|^{4 /(3+\varepsilon)} d x d s<\infty
$$

We recall that a Leray weak solution of the Navier-Stokes equations is a solution which is bounded and weakly continuous in the space of periodic divergence-free $L^{2}$ functions, whose gradient is square-integrable in space and time and which satisfies the energy inequality. Therefore, the fact that its curl is, for almost all $t$, in $L^{2}$ in space follows from the property of being a Leray solution. The boundedness of the spatial $L^{1}$ norm of the curl and the finiteness of the space and time $L^{4 /(3+\varepsilon)}$ norm of the second derivatives of the solution follow from the requirements that $\omega_{0}$ be a finite Radon measure and $u_{0}$ be an $L^{2}$ function. No assumption of smallness is needed. Expressed in terms of $\omega_{0}$ alone, the assumptions are that $\omega_{0}$ be a finite Radon measure which belongs to the space $V^{\prime}$, the dual of the space $V$ of periodic, divergence-free functions in $L^{2}$ which have periodic, square-integrable gradients. In particular, Theorem 2.2 is applicable for vortex sheet initial data of arbitrary strengths and actually, vortex lines and even much more singular vortex structures. In the case of small initial data and $F=0$ there are results in the literature $([\mathrm{G}-\mathrm{M}])$, concerning singular initial vorticities; if the initial vorticity is a Radon measure of small size belonging, moreover, to the Morrey class of order $\frac{3}{2}$ which, very roughly speaking corresponds to vortex line initial data, then there exist small global smooth solutions which decay in time. (See also [C-S] for a similar result.)

Under no other assumption than $u_{0}$ in $L^{2}$ weak Leray solutions satisfy certain estimates regarding high order derivatives $([F-G-T])$. For instance the $\frac{2}{3}$ power of the $H^{2}$ spatial norm has a priori bounded time average. (See [D] for similar results.)

The proof of Theorem 2.2 is somewhat laborious and therefore we will present here only the main ideas. We approximate the Navier-Stokes equations by a sequence of equations

$$
\begin{gathered}
\left(\frac{\partial}{\partial t}-v \Delta+S_{\delta}(v) \cdot \nabla\right) v+\nabla p=S_{\delta}(F) \\
\operatorname{div} v=0 \\
v(x, 0)=u_{0, \delta}
\end{gathered}
$$

where $\delta$ is a small number and $S_{\delta}(v), S_{\delta}(F)$ are obtained from $v, F$ by a convolution

$$
S_{\delta}(v)(x, t)=\delta^{-4} \int_{-\infty}^{t / T} \int_{\mathbb{R}^{3}} \psi\left(\frac{y}{\delta}, \frac{s}{\delta}\right) v(x-L y, t-T s) d y d s
$$

The smooth function $\psi$ is assumed to be non-negative with compact support in $|y|<1,1<s<2$ and have space and time integral equal to one. Note that if $v$ is space periodic with period $L$, so is $S_{\delta}(v)$. Moreover, the values of $S_{\delta}(v)$ at time $t$ depend only on the values of $v$ at positive times in the interval $(t-2 \delta T, t-\delta T)$. 
Also, clearly, if $v$ is divergence-free, so is $S_{\delta}(v)$. The initial data are also mollified

$$
u_{0, \delta}(x)=\delta^{-3} \int_{\mathbb{R}^{3}} \phi\left(\frac{y}{\delta}\right) u_{0}(x-L y) d y,
$$

where the smooth function $\phi$ is non-negative and has space integral equal to one. This procedure of constructing weak solutions is well-known. It was used in [C-K-N] to construct suitable weak solutions (that is solutions which obey a local energy estimate) of the Navier-Stokes equations. Thus, incidentally, the solutions that we construct are suitable weak Leray solutions and consequently, the space-time 1 dimensional Hausdorff measure of their singular set is zero $[\mathrm{C}-\mathrm{K}-\mathrm{N}]$. Most of the details of this proof which do not concern the new a priori bounds can be adapted directly for the periodic case from the appendix of $[\mathrm{C}-\mathrm{K}-\mathrm{N}]$. The solution to (2.23)-(2.25) is unique, global and smooth if $\delta$ is fixed and small enough. This can be proved using a standard Galerkin approximation of (2.23) and the fact that $S_{\delta}$ is smoothing. Moreover, from energy estimates of the solution $v$ of $(2.23)$ it follows that

and

$$
\sup _{0 \leqq t \leqq T} \int|v(x, t)|^{2} d x
$$

$$
\int_{0}^{T} \int|\nabla v(x, s)|^{2} d x d s
$$

are uniformly bounded, independently of $\delta$ for small $\delta$. The time derivatives of the solutions $v$ are uniformly bounded in $L^{4 / 3}\left(0, T ; V^{\prime}\right)$. Therefore by a well-known result (see, for instance, $[\mathrm{C}-\mathrm{F}],[\mathrm{T}]$ ) the $v$-s belong to a compact set in $L^{2}$ in space and time. These are standard results, and would have been true for other approximation methods, such as, for instance, the usual Galerkin method. We chose the retarded mollification approximation because the curls of the $v$ can be bounded uniformly in $L^{1}$. Indeed the equation for $\omega_{\delta}=\operatorname{curl} v$ is

$$
\left(\frac{\partial}{\partial t}-v \Delta+S_{\delta}(v) \cdot \nabla\right) \omega_{\delta}=S_{\delta}(f)-\varepsilon_{\cdot j k} S_{\delta}\left(\frac{\partial v_{l}}{\partial x_{j}}\right) \frac{\partial v_{k}}{\partial x_{l}}
$$

where $\varepsilon_{i j k}$ is the usual antisymmetric tensor. The right-hand side of (2.26) is uniformly bounded, independently of $\delta$ in $L^{1}$ in space and time. Moreover, the initial data for (2.26) are uniformly bounded, independently of $\delta$ in $L^{1}$ in space. Because the solutions $v$ are smooth we are allowed to perform the change to $w_{\delta}$, where $w_{\delta}=q\left(\tau \omega_{\delta}\right)$ and $q$ is defined in (2.16). We deduce that

and

$$
\sup _{t<T} \int\left|\omega_{\delta}(x, t)\right| d x
$$

$$
\int_{0}^{T} \int\left|\nabla \omega_{\delta}(x, s)\right|^{4 /(3+\varepsilon)} d x d s
$$

are uniformly bounded, independently of $\delta$. Consequently, these uniform bounds will be inherited by the weak limit.

We end this section with a few comments. Because uniqueness of Leray weak solutions is not known, the question arises whether weak solutions obtained by 
different approximation procedures from the same initial data enjoy the properties of the solution obtained in Theorem 2.2.

It would be intersting if a concrete characterization of the class of initial vorticities allowed by Theorem 2.2 would be available. While vortons are too singular even for this problem, dimensional analysis suggests that vorticities which are concentrated on certain sets of Hausdorff dimension $\geqq \frac{1}{2}$ and are smooth with respect to $\frac{1}{2}$-dimensional Hausdorff measure would be in this class.

If the initial velocities belong to $B V$, are square-integrable and divergence-free then Theorem 2.2 is applicable but it does not guarantee that the $B V$ norm of the solution is bounded a priori in time. However, the $L^{\infty}$ bound in time for the $B V$ norm of the solution fails logarithmically. By this we mean that one can prove that

$$
\int_{0}^{T} \exp \left(c \int|\nabla u(x, s)| d x\right) d s
$$

is a priori bounded for a certain $c>0$. The proof of this statement uses the fact $([\mathrm{F}-\mathrm{G}-\mathrm{T}])$ that

$$
\int_{0}^{T}\|\nabla u(\cdot, s)\|_{L^{\infty}}^{1 / 2} d s
$$

is bounded a priori and the fact that, because the operator $\omega \rightarrow \nabla u$ is of weak-type $(1,1)$ one has a logarithmic extrapolation inequality

$$
\int|\nabla u(x)| d x \leqq \tau L^{3}+c_{1}\left(\log _{+}\left(\frac{\|\nabla u\|_{L^{x}}}{\tau}\right)\right) \int|\omega(x)| d x .
$$

In the above inequality $\tau>0$ is arbitrary and $c_{1}$ is independent of $\tau$ and is the weak-type $(1,1)$ norm of the map $\omega \rightarrow \nabla u$. The constant $c$ above is related to $c_{1}$ by $c=\left(2 c_{1}\right)^{-1}$. The proof of (2.29) is simple. Denoting $N=\|\nabla u\|_{L^{\infty}}$ we have

$$
\int|\nabla u(x)| d x=\int_{0}^{N} \operatorname{meas}\{x ;|\nabla u(x)| \geqq \alpha\} d \alpha .
$$

If $\tau>N$ then clearly (2.29) is true. If $\tau<N$ then we split the integral above in two parts. The first part we bound

$$
\int_{0}^{\tau} \operatorname{meas}\{x ;|\nabla u(x)| \geqq \alpha\} d \alpha \leqq \tau L^{3},
$$

and in the second part we use the weak-type inequality

$$
\operatorname{meas}\{x ;|\nabla u(x)| \geqq \alpha\} \leqq c_{1}\|\omega\|_{L^{1}} \alpha^{-1}
$$

to bound

$$
\left.\int_{\tau}^{N} \operatorname{meas}\{x ;|\nabla u(x)|) \geqq \alpha\right\} d \alpha \leqq c_{1}\|\omega\|_{L^{1}} \log \left(\frac{N}{\tau}\right) .
$$

As $\tau$ is arbitrary we may choose in (2.29) $\tau=\|\omega\|_{L^{1}} L^{-3}$. The a priori bound of (2.27) follows using (2.29) multiplied by $\left(2 c_{1}\right)^{-1}$ and exponentiated, the bound on (2.28) and Theorem 2.2. Strictly speaking these operations need to be done for each approximate solution $v$, but the argument remains the same. 


\section{The Area of Level Sets of the Vorticity}

In this section we will consider the problem of estimating a priori in terms of the Reynolds number the typical area of a level set of the vorticity magnitude. We consider an arbitrary solution of the Navier-Stokes equations (2.1)-(2.4). For any positive number $\gamma$ we consider the level sets

$$
A_{\gamma}=\{x ;|\omega(x, t)|=\gamma\} .
$$

If $\omega$ is a smooth function then it is well-known ([M]) that for almost all $\gamma$ the sets $A_{\gamma}$ are smooth 2-dimensional manifolds without boundary in $T_{L}^{3}$. We denote their 2-dimensional Hausdorff measure (area in this case) by $\mu(\gamma, t)$ :

$$
\mu(\gamma, t)=\int_{A_{\gamma}} d H^{(2)}(x) .
$$

We are interested in estimating the average $\langle\mu\rangle$ in terms of the Reynolds number. Our method works also for local averages, that is averages of the area of portions of level sets confined to some fixed region of space. Also, the fact that we restrict ourselves to space periodic and body driven flows is not essential.

In the vorticity equation (2.5) we perform the change of dependent variable (2.8) with $q$ given in (2.13). The number $\tau$ is a convenient time scale and $h(\alpha)=\tau^{-1} \alpha^{1 / 2}$. Thus we consider the function

$$
w(x, t)=\left(\tau^{-2}+|\omega(x, t)|^{2}\right)^{1 / 2} .
$$

Then $w$ satisfies (2.9) with $R$ bounded independently of $\tau$ :

$$
|R| \leqq|\omega||\nabla u|+|f| .
$$

Moreover, direct computation of (2.14) shows that

$$
\left(\frac{\partial}{\partial t}+u \cdot \nabla u-v \Delta\right) w \leqq|\omega||\nabla u|+|f| .
$$

The sets $A_{\gamma}$ are level sets of $w$ :

$$
A_{\gamma}=\left\{x ; w(x, t)=\left(\tau^{-2}+\gamma^{2}\right)^{1 / 2}\right\} .
$$

We take a positive, increasing function of one variable $G(\beta)$ and multiply the inequality (3.5) by $G^{\prime}(w(x, t))$, where $G^{\prime}$ is the derivative of $G$. We obtain

$$
\left(\frac{\partial}{\partial t}+u \cdot \nabla-v \Delta\right) g+v G^{\prime \prime}|\nabla w|^{2} \leqq|R| G^{\prime}
$$

In (3.7) we denoted

$$
g(x, t)=G(w(x, t)),
$$

and $G^{\prime}, G^{\prime \prime}$ were computed at $w(x, t)$. Integrating in (3.7) we obtain

$$
v \int_{0}^{T} \int G^{\prime \prime}|\nabla w|^{2} d x d t \leqq \int_{0}^{T} \int G^{\prime}|R| d x d t+\int(g(x, 0)-g(x, T)) d x .
$$

We will specify $G$ in the following way. We take an arbitrary smooth function 
of one variable $\phi(\beta)$ compactly supported in the interval $\left(\tau^{-1}, \infty\right)$ and define

$$
G(\beta)=\int_{\tau}^{\beta}(\beta-\lambda) \phi^{2}(\lambda) d \hat{\lambda}
$$

We observe that

$$
0 \leqq G(\beta) \leqq \beta\|\phi\|^{2}
$$

where

$$
\|\phi\|^{2}=\int_{-\infty}^{\infty} \phi^{2}(\hat{\lambda}) d \lambda
$$

and

$$
G^{\prime}(\beta)=\int_{\tau-1}^{\beta} \phi^{2}(\lambda) d \lambda
$$

Consequently,

$$
0 \leqq G^{\prime}(\beta) \leqq\|\phi\|^{2}
$$

Moreover,

$$
G^{\prime \prime}(\beta)=\phi^{2}(\beta) .
$$

Using (3.10)-(3.15) in (3.9) we obtain

$$
v \int_{0}^{T} \int \phi^{2}(w(x, t))|\nabla w(x, t)|^{2} d x d t \leqq\|\phi\|^{2}\left(\int_{0}^{T} \int|R| d x d t+\int w(x, 0) d x\right),
$$

and, as a consequence

$$
T^{-1} \int_{0}^{T} \int \phi(w(x, t))|\nabla w(x, t)| d x d t \leqq\|\phi\| \mathscr{E}(T)
$$

where

$$
\mathscr{E}(T)=v^{-1 / 2} L^{3 / 2}\left(T^{-1} \int_{0}^{T} \int|R| d x d t+T^{-1} \int w(x, 0) d x\right)^{1 / 2} .
$$

Now we will use an important tool of geometric measure theory, established by Kronrod $([\mathrm{K}])$ in a speciål case and by Federer $([\mathrm{F}])$ in the general case. A proof can be found in $[\mathrm{M}]$ also.

Theorem 3.1. Let $\psi$ be a Borel measurable nonnegative function on $D$ and let $h$ be a Lipschitz continuous function in $D$, where $D$ is an open subset of $\mathbb{R}^{n}$. Then

$$
\int_{D} \psi(x)|\nabla h(x)| d x=\int_{0}^{\infty} d \beta \int_{\mathscr{A}_{\beta}} \psi(x) d H^{(n-1)}(x),
$$

where $H^{(n-1)}$ is the $(n-1)$-dimensional Hausdorff measure and

$$
\mathscr{A}_{\beta}=\{x \in D ;|h(x)|=\beta\} \text {. }
$$

Theorem 3.1 is local and therefore it is valid for periodic functions, also. We 
will use it in (3.17), with $h=w$ and $\psi(x)=\phi(w(x, t))$. We obtain

$$
T^{-1} \int_{0}^{T} d t \int_{\tau^{-1}}^{\infty} \phi(\beta) d \beta \int_{\mathscr{A}_{\beta}} d H^{(2)}(x) \leqq\|\phi\| \mathscr{E}(T) .
$$

Now, in view of (3.1), (3.6) and (3.20) we deduce

$$
\mathscr{A}_{\beta}=A_{\left(\beta^{2}-\tau^{-2}\right)^{1 / 2}}
$$

and therefore the inequality (3.21) can be re-written as

$$
T^{-1} \int_{0}^{T} \int_{\tau}^{\infty} \phi(\beta) \mu\left(\left(\beta^{2}-\tau^{-2}\right)^{1 / 2}, t\right) d \beta d t \leqq\|\phi\| \mathscr{E}(T) .
$$

Let us define $\mu_{T}$ by

$$
\mu_{T}(\gamma)=T^{-1} \int_{0}^{T} \mu(\gamma, t) d t
$$

Because $\phi$ is arbitrary we obtain from (3.23) that

$$
\int_{\tau^{-1}}^{\infty} \mu_{T}^{2}\left(\left(\beta^{2}-\tau^{-2}\right)^{1 / 2}\right) d \beta \leqq(\mathscr{E}(T))^{2}
$$

We perform the change of variable

$$
\left(\beta^{2}-\tau^{-2}\right)^{1 / 2}=\gamma
$$

in the left-hand side of (3.25). We obtain

$$
\int_{0}^{\infty} \mu_{T}^{2}(\gamma) \frac{\gamma}{\left(\gamma^{2}+\tau^{-2}\right)^{1 / 2}} d \gamma \leqq(\mathscr{E}(T))^{2}
$$

In view of the definition (3.18) of $\mathscr{E}(T)$ and (3.3) of $w$ we see that

$$
\lim _{\tau \rightarrow \infty} \mathscr{E}(T)=\mathscr{R}(T)
$$

with

$$
\mathscr{R}(T)=v^{-1 / 2} L^{3 / 2}\left(T^{-1} \int_{0}^{T} \int|R| d x d t+T^{-1} \int\left|\omega_{0}(x)\right| d x\right)^{1 / 2} .
$$

Passing to the limit of $\tau \rightarrow \infty$ we obtain the inequality

$$
\int_{0}^{\infty} \mu_{T}^{2}(\gamma) d \gamma \leqq(\mathscr{R}(T))^{2}
$$

In a completely analogous fashion, passing to lim sup in (3.23) we obtain

$$
T \rightarrow \infty
$$

where

$$
\int_{0}^{\infty} \mu_{\infty}^{2}(\gamma) d \gamma \leqq \mathscr{R}_{\infty}^{2}
$$

$$
\mu_{\infty}(\gamma)=\limsup _{T \rightarrow \infty} T^{-1} \int_{0}^{T} \mu(\gamma, t) d t
$$


and

$$
\mathscr{R}_{\infty}^{2}=v^{-1} L^{3} \limsup _{T \rightarrow \infty} T^{-1} \int_{0}^{T} \int|R| d x d t .
$$

In view of the inequality (3.4) we proved the following theorem:

Theorem 3.2. Let $\omega$ be the curl of a classical $\left(\mathscr{C}^{3}\right)$ L-periodic solution of the Navier-Stokes equation (2.1) with smooth periodic driving forces $F$. Assume that the solution is defined for $0 \leqq t \leqq T_{1}$, where $T_{1} \leqq \infty$. Then the area

$$
\mu(\gamma, t)=\int_{\{x ;|\omega(x, t)|=\gamma\}} d H^{(2)}(x)
$$

satisfies, for any $0<T<T_{1}$,

$$
\int_{0}^{\infty} \mu_{T}^{2}(\gamma) d \gamma \leqq v^{-1} L^{3} T^{-1}\left(\int_{0}^{T} \int\left(|\omega(x, t)|^{2}+|\operatorname{curl} F(x, t)|\right) d x d t+\int\left|\omega_{0}(x)\right| d x\right),
$$

where $\mu_{T}$ is the time average of $\mu$ defined in (3.24). Moreover, if $T_{1}=\infty$ then

$$
\int_{0}^{\infty} \mu_{\infty}^{2}(\gamma) d \gamma \leqq v^{-1} L^{3} \limsup _{T \rightarrow \infty} T^{-1} \int_{0}^{T} \int\left(|\omega(x, t)|^{2}+|\operatorname{curl} F(x, t)|\right) d x d t,
$$

where $\mu_{\infty}=\limsup _{T \rightarrow \infty} \mu_{T}$

In view of the energy inequality (2.21) the inequalities (3.35) and (3.36) imply the a priori bounds in terms of initial data and $F$

$$
\begin{aligned}
\int_{0}^{\infty} \mu_{T}^{2}(\gamma) d \gamma \leqq & v^{-2} L^{3} T^{-1} \int\left|u_{0}(x)\right|^{2} d x+v^{-1} L^{3} T^{-1} \int\left|\omega_{0}(x)\right| d x \\
& +v^{-3} L^{5} T^{-1} \int_{0}^{T} \int|F(x, t)|^{2} d x d t+v^{-1} L^{3} T^{-1} \int_{0}^{T} \int|\operatorname{curl} F(x, t)| d x d t,
\end{aligned}
$$

and

$$
\int_{0}^{\infty} \mu_{\infty}^{2}(\gamma) d \gamma \leqq v^{-1} L^{3} \limsup _{T \rightarrow \infty} T^{-1} \int_{0}^{T} \int\left(L^{2} v^{-2}|F(x, t)|^{2}+|\operatorname{curl} F(x, t)|\right) d x d t .
$$

Note that the right-hand sides of (3.35)-(3.38) are finite even if the solution becomes singular.

Both the left-hand and right-hand sides of (3.35)-(3.38) have the dimension $\langle L\rangle^{4}\langle T\rangle^{-1}$. The left-hand sides have this dimension because $\mu$ represents an area and $\gamma$ is a vorticity magnitude and thus scales like $\langle T\rangle^{-1}$.

We define average values of $\mu$ by the formulas

$$
\langle\mu\rangle_{T}=\left([\omega]_{T}^{-1} \int_{0}^{\infty} \mu_{T}^{2}(\gamma) d \gamma\right)^{1 / 2}
$$

and

$$
\langle\mu\rangle_{\infty}=\left([\omega]_{\infty}^{-1} \int_{0}^{\infty} \mu_{\infty}^{2}(\gamma) d \gamma\right)^{1 / 2},
$$


where

$$
[\omega]_{T}=\sup _{0 \leqq t \leqq T} \sup _{x}|\omega(x, t)|
$$

and

$$
[\omega]_{\infty}=\sup _{0 \leqq t \leqq \infty} \sup _{x}|\omega(x, t)| .
$$

These definitions are natural; the reason for dividing by the numbers defined in (3.41), (3.42) is that, for smooth solutions, the range of $\gamma$ is $[0,[\omega]]$ and not $[0, \infty)$. We emphasise that the numbers defined in (3.39) and (3.40) do not involve any ensemble averaging. They are well defined for any individual smooth, deterministic solution of the Navier-Stokes equations and represent, in some sense, an average value of the area of vorticity level sets.

Let us consider now the particular case of $F=0$. Then (3.35) becomes

$$
\langle\mu\rangle_{T}^{2} \leqq v^{-1} L^{6}\left(\sqrt{\frac{\varepsilon}{v}}+T^{-1}\right),
$$

where the energy dissipation rate is given by

$$
\varepsilon=v T^{-1} L^{-3} \int_{0}^{T} \int|\omega(x, t)|^{2} d x d t
$$

We define the Reynolds number Re by

$$
\operatorname{Re}=T^{2} \varepsilon v^{-1}
$$

(see [B], (7.3.3)). One can think of (3.45) as defining, for fixed Reynolds number, a time $T$ which is large enough for turbulence to reach statistical universality but which is smaller than the viscous dissipation time scale $L^{2} v^{-1}$. We recall that the Kolmogorov dissipation length $\eta$ is defined by

$$
\eta=\varepsilon^{-1 / 4} v^{3 / 4}
$$

The inequality (3.43) becomes

$$
L^{-2}\langle\mu\rangle_{T} \leqq \frac{L}{\eta}\left(1+\mathrm{Re}^{-1 / 2}\right)^{1 / 2}
$$

or, because

$$
\begin{gathered}
\eta=\varepsilon^{1 / 2} T^{3 / 2} \operatorname{Re}^{-3 / 4}, \\
L^{-2}\langle\mu\rangle_{T} \leqq \operatorname{Re}^{3 / 4} L \varepsilon^{-1 / 2} T^{-3 / 2}\left(1+\operatorname{Re}^{-1 / 2}\right)^{1 / 2} .
\end{gathered}
$$

The inequality (3.47) can be re-written as

$$
\langle\mu\rangle_{T} \leqq C\left(\frac{L}{\eta}\right)^{3} \eta^{2}
$$

with $C=\left(1+\mathrm{Re}^{-1 / 2}\right)^{1 / 2}$. The right-hand side of (3.50) has a simple geometric interpretation. It is proportional with the area of a union of non-overlapping spheres of radii $\eta$ which fills the cube of side length $L$.

We proved thus 
Theorem 3.3. The average area $\langle\mu\rangle_{T}$ of a level set of the vorticity magnitude of $a$ smooth solution of the Navier-Stokes equation (2.1) with $F=0$ is bounded above by a multiple of $\mathrm{Re}^{3 / 4}$, where $\mathrm{Re}$ is the Reynolds number. When expressed in terms of the Kolmogorov dissipation length $\eta$ this upper bound ((3.50)) represents the area of a union of non-overlapping spheres of radii $\eta$ which fill a fraction $\left.(1 / 4 \pi) \sqrt{1+\operatorname{Re}^{-1 / 2}}\right)$ of the volume of the spatial domain.

\section{Area of Isotherms}

We investigate the problem of estimating a priori the average area of portions of level sets of scalars. As an application we will consider the area of portions of isotherms in Rayleigh-Benard convection.

We consider a real scalar function $T(x, t)$ defined for $x$ in a bounded, open domain $D$ of $\mathbb{R}^{3}$ with smooth boundary and $t$ nonnegative. We assume that $T$ satisfies the equation

$$
\left(\frac{\partial}{\partial t}+u \cdot \nabla-\kappa \Delta\right) T=0
$$

in $D$ where $\kappa$ is a positive constant and the $\mathbb{R}^{3}$ valued function $u$ satisfies

$$
\operatorname{div} u=0
$$

and

$$
u_{\mid \partial D}=0 \text {. }
$$

In the case of Rayleigh-Benard convection $u$ satisfies a partial differential equation which couples its evolution with that of $T$. We will study the area of level sets of $T$ and bound them in terms of certain average bounds on $u$. At this stage we do not make more assumptions on $u$ than (4.2), (4.3) and the minimal regularity assumptions needed to solve (4.1). We supplement (4.1) with initial data

$$
T(x, 0)=T_{0}(x)
$$

We assume that $T_{0}$ is bounded. We will not specify boundary conditions on $T$. Rather, we will assume a maximum principle:

$$
0 \leqq T(x, t) \leqq M
$$

with $M>0$ a given number. The inequality (4.5) is consequence of standard boundary conditions on $T$. In particular, the usual boundary conditions in convection, $T=0$ on the top plate, $T=M$ on the bottom one and either Neumann or periodic boundary conditions on the lateral walls imply (4.5) provided, of course, that $T_{0}$ obeys (4.5). Thus (4.5) does not impose any restriction if $T$ satisfies one of these standard boundary conditions; in many geophysical problems, however, the boundary conditions are somewhat unclear and therefore it seems worthwhile to make minimal assumptions on the boundary behavior of $T$. We take a smooth, compactly supported, nonnegative function $\psi$ whose support is at distance $\geqq \delta>0$ from the boundary of $D$. We take also the function $G$ defined in (3.10) except that 
now we can set already $\tau=\infty$. Thus

$$
G(\beta)=\int_{0}^{\beta}(\beta-\lambda) \phi^{2}(\lambda) d \lambda
$$

with $\phi$ an arbitrary smooth function supported in $(0, \infty)$. We multiply (4.1) by $\psi(x) G^{\prime}(T(x, t))$. We obtain

$$
\left(\frac{\partial}{\partial t}+u \cdot \nabla-\kappa\right) g_{\psi}+\kappa \psi G^{\prime \prime}|\nabla T|^{2}=R
$$

where

$$
g_{\psi}(x, t)=\psi(x) G(T(x, t))
$$

the derivative $G^{\prime \prime}$ is computed at $T(x, t)$ and $R$ is given by

$$
R=G(T)(u \cdot \nabla \psi-\kappa \Delta \psi)-2 \kappa G^{\prime}(T) \nabla T \cdot \nabla \psi .
$$

Integrating over $D$ we obtain

$$
\frac{d}{d t} \int_{D} g_{\psi}(x, t) d x+\kappa \int_{D} \psi(x) G^{\prime \prime}(T(x, t))|\nabla T(x, t)|^{2} d x=\mathscr{R}(t)
$$

with

$$
\mathscr{R}(t)=\int_{D} G(T(x, t))(u \cdot \nabla \psi+\kappa \Delta \psi)(x, t) d x .
$$

The relations (3.11)-(3.15) regarding $G$ are valid. It follows that

$$
\mathscr{R}(t) \leqq\|\phi\|^{2} M \int_{D}|u \cdot \nabla \psi+\kappa \Delta \psi| d x
$$

Integrating (4.10) in time we deduce

$$
\int_{0}^{t} \int_{D} \psi(x) \phi^{2}(T(x, s))|\nabla T(x, s)|^{2} d x d s=\kappa^{-1}\|\phi\|^{2} E(t)
$$

with

$$
\|\phi\|^{2} E(t)=\int_{D}\left(g_{\psi}(x, 0)-g_{\psi}(x, t)\right) d x+\int_{0}^{t} \mathscr{R}(s) d s .
$$

It follows that

$$
t^{-1} \int_{0}^{t} \int_{D} \psi(x) \phi(T(x, s))|\nabla T(x, s)| d x d s \leqq\|\phi\|\left(\kappa^{-1} t^{-1} E(t) \int_{D} \psi(x) d x\right)^{1 / 2} .
$$

Using Theorem 3.1 it follows that the left-hand side of (4.15) equals

$$
\int_{0}^{M} \phi(\beta)\left(t^{-1} \int_{0}^{t} \mu_{\psi}(\beta, s) d s\right) d \beta
$$

where

$$
\mu_{\psi}(\beta, s)=\int_{\{x ; T(x, s)=\beta\}} \psi(x) d H^{(2)}(x)
$$


Let us denote the time average of $\mu_{\psi}$ by $\mu_{\psi, t}$ :

$$
\mu_{\psi, t}(\beta)=t^{-1} \int_{0}^{t} \mu_{\psi}(\beta, s) d s
$$

Then, because $\phi$ is arbitrary in (4.15) it follows that

$$
\int_{0}^{M} \mu_{\psi, t}^{2}(\beta) d \beta \leqq \kappa^{-1} t^{-1}\left(\sup _{\|\phi\| \leqq 1} E(t)\right) \int_{D} \psi(x) d x .
$$

Now it is time to choose $\psi$. There are several types of choices, depending on the region in $D$ we want to investigate. We will present two choices: the first one is suited for the situation in which the average value of $\left|u\left(x_{0}, t\right)\right|$ is measured at a fixed $x_{0}$ in $D$ while the second one regards behavior in the whole of $D$ minus a thin layer around the boundary.

Let us take a nonnegative smooth function $\psi_{0}$ which is identically equal to one in $\{y ;|y| \leqq 1\}$ and vanishes identically for $|y| \geqq 2$. Let $x_{0} \in D$ be fixed and set

$$
\psi(x)=\psi_{0}\left(\frac{x-x_{0}}{r}\right)
$$

where $r>0$ is a small number. Clearly, we have

$$
\begin{gathered}
|\psi(x)| \leqq C_{1}, \\
|\nabla \psi(x)| \leqq C_{2} r^{-1},
\end{gathered}
$$

and

$$
|\Delta \psi(x)|=C_{3} r^{-2}
$$

for some fixed $C_{1}, C_{2}, C_{3}$ and all $x$. It follows from (4.19) the definition (4.14) of $E(t)$ and (4.2) that

$$
\tau^{-1} \int_{0}^{M} \mu_{\psi, t}^{2}(\beta) d \beta \leqq C^{2} \kappa^{-1}\left(r^{2} t^{-1} \int_{0}^{t} \int_{B\left(x_{0}, 2 r\right)}|u(x, s)| d s d t+\kappa r^{4}+\sigma r^{5}\right),
$$

where we denoted by $B\left(x_{0}, 2 r\right)$ the ball of radius $2 r$ centered at $x_{0}, C$ is a constant $\tau$ is the oscillation of $T$ :

$$
\tau=\sup _{x, y \in B\left(x_{0}, 2 r\right), 0 \leqq s \leqq t}|T(x, s)-T(y, s)|,
$$

and $\sigma$ is given by

$$
\sigma=r t^{-1}\left(2+\tau^{-1} \sup _{0 \leqq s \leqq t}\left(T\left(x_{0}, 0\right)-T\left(x_{0}, s\right)\right)_{+}\right) .
$$

Now, because $\psi=1$ on the ball $B\left(x_{0}, r\right)$ it follows that

$$
\tau^{-1} \int_{0}^{M} \mu_{r, t}^{2}(\beta) d \beta \leqq C^{2} \kappa^{-1}\left(r^{5}\left[|u|_{2 r, t}\left(x_{0}\right)+\sigma\right]+\kappa r^{4}\right),
$$

where

$$
\mu_{r, t}(\beta)=t^{-1} \int_{0}^{t} d s \int_{\left\{x ;\left|x-x_{0}\right| \leqq r, T(x, s)=\beta\right\}} d H^{(2)}(x)
$$


and

$$
|u|_{2 r, t}\left(x_{0}\right)=\frac{2}{\pi} t^{-1} r^{-3} \int_{0}^{t} \int_{B\left(x_{0}, 2 r\right)}|u(x, s)| d x d s .
$$

Let us assume, for the same of clarity, that $u(\cdot, s)$ is continuous at $x_{0}$ for all $s$ under consideration. This assumption is not needed: if $u(\cdot, s)$ is locally in $L^{p}$ then the Hardy-Littlewood maximal function computed at $x_{0}$ and averaged in time majorizes $|u|_{2 r, t}\left(x_{0}\right)$. We define $\langle\mu\rangle_{r, t}$ by

$$
\langle\mu\rangle_{r, t}\left(x_{0}\right)=\left(\tau^{-1} \int_{0}^{M} \mu_{r, t}^{2}(\beta) d \beta\right)^{1 / 2} .
$$

We would like to think of $\langle\mu\rangle_{r, t}\left(x_{0}\right)$ as representing an average value of the area of the piece of a surface $T=$ constant contained in $B\left(x_{0}, r\right)$. We deduce from (4.26)

Theorem 4.1. Let $T$ be a solution of (4.1) and $x_{0}$ a fixed point in $D$.

Then the average area $\langle\mu\rangle_{r, t}\left(x_{0}\right)$ of a piece of a surface $T=$ constant contained in a ball of radius $r$ centered at $x_{0}$ is bounded by

$$
\langle\mu\rangle_{r, t}\left(x_{0}\right) \leqq C \kappa^{-1 / 2} r^{5 / 2}\left(|u|_{2 r, t}\left(x_{0}\right)+\sigma\right)^{1 / 2}+C r^{2}
$$

for small enough $r$.

Note that although both sides of (4.30) have the correct dimension of area, (4.30) would suggest that the local (ensemble) average level set of $T$ has fractal dimension larger or equal than 2.5. It is perhaps significant to note that experimental evidence ([S]) seems to indicate that 2.35 is a lower bound for this dimension.

Let us consider the Hardy-Littlewood maximal function

$$
\langle|u|(x)\rangle=\sup _{0<r \leqq \delta} c r^{-3} \int_{B(x, 2 r)} t^{-1} \int_{0}^{t}|u(y, s)| d s d y
$$

and associate to it the local length scale

$$
\lambda=\kappa\langle|u|(x)\rangle^{-1} .
$$

Then in the right-hand side of (4.30) the term involving $r^{2.5}$ is larger than the one involving $r^{2}$ for $r$ small but $r \geqq \lambda$. Now $\lambda$ is typically a very small number, or in other words the coefficient of $r^{2}$ in (4.30) is very small in comparison to the coefficient of $r^{2.5}$. The main point is that in the case of turbulent flows $\lambda$ is smaller than the smallest physically significant scale. For instance, if $u$ has the statistics given by a homogeneous self-similar family of statistical solutions of the Navier-Stokes equations ([FMT]) and one identifies $\langle|u|\rangle$ with the average velocity magnitude then

$$
\lambda=\operatorname{Pr}^{-1} \operatorname{Re}^{-1 / 4} \eta
$$

(C. Foias, private communication). Or, if one considers solutions of the Boussinesq approximation for Rayleigh-Benard convection and identities the quantity $\langle|u|\rangle$ with $u_{c}$ (the velocity in the central region) one obtains

$$
\lambda=L \mathrm{Ra}^{-1+2 \beta}
$$


where $L \mathrm{Ra}^{-\beta}$ represents the width of the thermal boundary layer (see [Chi], (3.5)-(3.7)). In the classical $\left(\beta=\frac{1}{3}\right)$ regime one deduces that $\lambda$ equals precisely the width of the thermal boundary layer. In the hard turbulence $\left(\beta=\frac{2}{7}\right)$ regime $\lambda$ is smaller than the boundary layer width. Thus, (4.30) seems to indicate that if one is not allowed to go beyond the smallest physically significant scale then the average local area of isotherms scales like $r^{2.5}$.

Now we will consider the problem of estimating the average area of a level set of $T$ contained in $D_{\delta}$, the set of points in $D$ situated at a distance of at least $\delta$ from the boundary of $D$. Taking the characteristic function of $D_{\delta / 2}$ and using a standard mollifier we can find a smooth, nonnegative $\psi$ which is identically equal to one in $D_{\delta}$, has compact support in $D$ and satisfies

$$
\begin{gathered}
\psi(x) \leqq C_{1}, \\
|\nabla \psi(x)| \leqq C_{2} \delta^{-1},
\end{gathered}
$$

and

$$
|\Delta \psi(x)| \leqq C_{3} \delta^{-2}
$$

We will assume, for simplicity, that $D$ has aspect ratio equal to one and diameter $L$.

Using the inequalities (4.34)-(4.36) in (4.19) we obtain

$$
M^{-1} \int_{0}^{M} \mu_{\psi, t}^{2}(\beta) d \beta \leqq C^{2} \kappa^{-1} L^{3}\left(\delta^{-1} t^{-1} \int_{0}^{t} \int_{D}|u(x, s)| d x d s+\kappa \delta^{-2} L^{3}+t^{-1} L^{3}\right) .
$$

We define $\mu_{\delta, t}(\beta)$ by

$$
\mu_{\delta, t}(\beta)=t^{-1} \int_{0}^{t} d s \int_{\left\{x \in D_{\delta} ; T(x, x)=\beta\right\}} d H^{(2)}(x)
$$

and $\langle|u|\rangle$ by

$$
\langle|u|\rangle=t^{-1} L^{-3} \int_{0}^{t} \int_{D}|u(x, s)| d x d s .
$$

Then we deduce from (4.37),

$$
M^{-1} \int_{0}^{M} \mu_{\delta, t}^{2}(\beta) d \beta \leqq C^{2} \kappa^{-1} L^{6}\left(\delta^{-1}\langle|u|\rangle+\kappa \delta^{-2}+t^{-1}\right)
$$

Now we set

$$
\langle\mu\rangle_{\delta, t}=\left(M^{-1} \int_{0}^{M} \mu_{\delta, t}^{2}(\beta) d \beta\right)^{1 / 2},
$$

and conclude from (4.40) that

$$
\langle\mu\rangle_{\delta, t} \leqq C L^{3}\left(\kappa^{-1 / 2} \delta^{-1 / 2}\langle|u|\rangle^{1 / 2}+\delta^{-1}+\kappa^{-1 / 2} t^{-1 / 2}\right) .
$$

We proved thus

Theorem 4.2. The average area $\langle\mu\rangle_{\delta, t}$ of a piece of a surface $T=$ constant contained in $D_{\delta}$ satisfies (4.42). 
As an application let us consider the Boussinesq equations for Rayleigh-Benard convection. The Eq. (4.1) for $T$ is supplemented with

$$
\left(\frac{\partial}{\partial t}+u \cdot \nabla-v \Delta\right) u+\nabla p=g \alpha T e_{3} .
$$

Also (4.2)-(4.5) hold. In (4.43) $g$ is the accelaration of gravity, $\alpha$ the volume thermal expansion coefficient $e_{3}$ is the vertical direction and $v$ is the kinematic viscosity. The Rayleigh number is

$$
\mathrm{Ra}=\frac{\alpha g M L^{3}}{\kappa v} .
$$

We want to give an upper bound for $\langle|u|\rangle$ in terms of the Rayleigh number. We multiply scalarly (4.43) by $u$ and integrate in $D$. Using the Poincaré inequality we obtain

$$
\frac{d}{d t}\|u\|+v L^{-2}\|u\| \leqq g \alpha M L^{3 / 2}
$$

where

$$
\|u\|^{2}=\int_{D}|u(x, t)|^{2} d x
$$

Consequently

$$
\langle|u|\rangle \leqq \kappa L^{-1} \mathrm{Ra}+t^{-1} v^{-1} L^{1 / 2}\left\|u_{0}\right\| .
$$

Direct application of Theorem 4.2 yields

Theorem 4.3. The average area $\langle\mu\rangle_{\delta, t}$ of a piece of surface $T=$ constant contained in $D_{\delta}$ satisfies

$$
\langle\mu\rangle_{\delta, t} \leqq 2 C\left(L^{3} \delta^{-1}+L^{5 / 2} \delta^{-1 / 2} \mathrm{Ra}^{1 / 2}\right)
$$

for $t$ sufficiently large.

Acknowledgements. I thank C. Foias, L. Kadanoff and R. Temam for fruitful conversations.

\section{References}

[B] Batchelor, G. K.: The theory of homogeneous turbulence. Cambridge: Cambridge University Press

[C-F] Constantin, P., Foias, C.: Navier-Stokes Equations. Chicago, IL: The University of Chicago Press

[C-K-N] Caffarelli, L., Kohn, R., Nirenberg, L.: Partial regularity of suitable weak solutions of the Navier-Stokes equations. Commun. Pure Appl. Math. 35, 771-831 (1982)

[C-S] Cottet, G.H., Soler, J.: Three dimensional Navier-Stokes equations for singular filament initial data. J. Diff. Eqns 74, 234-253 (1988)

[Chi] Castaing, B. Gunaratne, G., Heslot, F., Kadanoff, L., Libchaber, A., Thomae, S., Wu, X-Z., Zaleski, S., Zanetti, G.: Scaling of hard thermal turbulence in Rayleigh Benard convection, preprint

[Cho] Chorin, A.: Scaling laws in the vorticity lattice model of turbulence. Commun. Math. Phys. 114, 167-176 (1988) 
[D] Duff, G. F. D.: Derivative estimates for the Navier-Stokes equations in a three dimensional region, preprint (1988)

[F] Federer, H.: Geometric measure theory. Berlin, Heidelberg, New York: Springer

[F-G-T] Foias, C., Guillope, C., Temam, R.: New a priori estimates for Navier-Stokes equations in dimension 3. Commun. P.D.E. 6, 329-359 (1981)

[F-M-T] Foias, C., Manley, O., Temam, R.: Self-similar invariant families of turbulent flows. Phys. Fluid 30, 2007-2020 (1987)

[Fo] Foias, C.: Private communication

[G-M] Giga, Y., Miyakawa, T.: Navier-Stokes flow in $\mathbb{R}^{3}$ with measures as initial vorticity and Morrey spaces, preprint (1988)

[K] Kronrod, A. S.: On functions of two variables. Usp. Math. Nauk. 5, 24-134 (1950) (Russian)

[L] Leray, J.: Sur le mouvement d'un liquide visqueux emplissant l'espace. Acta Math. 63, 193-248 (1934)

[M] Maz’ja, V. G.: Sobolev Spaces. Berlin, Heidelberg, New York: Springer

[S] Sreenivasan, K. R.: The physics of fully turbulent flows: Some recent contributions motivated by advances in dynamical systems, preprint (1989)

[T] Temam, R.: Navier-Stokes equations: theory and numerical analysis. Amsterdam, New York: North Holland

Communicated by S.-T. Yau

Received July 11, 1989; in revised form October 5, 1989 